\title{
INFLUÊNCIA DO MATERIAL DO BACKING NO FLUXO DE CALOR E NA FORMAÇÃO DE ZONAS DEFORMADAS PELO PROCESSO FSW EM LIGAS DE ALUMÍNIO
}

Marcio Jose Cuccolo Rosales ' Nelson Guedes de Alcântara ${ }^{2}$ Jorge Fernandez dos Santos ${ }^{3}$

\section{Resumo}

O desenvolvimento de novos materiais estruturais - especialmente ligas leves e aços de elevada resistência mecânica - vem sendo amplamente impulsionado pelas indústrias automotiva e aeroespacial. Embora sua aplicação seja muitas vezes limitada em função da dificuldade de unirem-se tais materiais, Friction Stir Welding (FSW) é um processo de soldagem no estado sólido que proporciona ótimo desempenho. Para o melhor controle do processo, este estudo analisa as condições térmicas (geração e fluxo de calor) e de deformação plástica das ligas do sistema $\mathrm{Al}-\mathrm{Mg}-\mathrm{Si}$ e Al-Cu-Mg, por meio do uso de três diferentes materiais que compõem o backing bar (cobre, aço e cerâmico). Além de influenciar a transferência de calor e impedir a perda de material na junta, o backing contribui para alterações microestruturais e a consolidação do material na raiz da solda. $O$ presente trabalho visa relacionar a influência do backing na formação de zonas deformadas pelo processo FSW, a fim de compreender a transferência de calor em relação às variáveis do processo. A extração e o fluxo de calor variam em função da condutividade do backing e da resistência à deformação plástica dos materiais.

Palavras-chave: Friction stir welding; Backing bar; Ligas de alumínio; Transferência de calor.

\section{FRICTION STIR WELGING OF ALUMINIUM ALLOYS}

\begin{abstract}
Although new structural and advanced materials are being considered for use in the automotive and aircraft industries, especially lightweight alloys as well as advanced high strength steels, the successful introduction of such materials depends on the availability of proven joining technologies that can provide for high quality and performance joints. Solid-state joining techniques such as Friction Stir Welding (FSW) are a natural choice since welds are produced at low temperatures thus the low heat input provides for limited distortion; and microstructural as well as mechanical degradation. Great effort is currently being made in the joining of Al-Cu-Mg and lately in the Al-Mg-Si alloys because of their high strength, improved formability and application in airframe structures. The backing bar plays an important role in heat transfer from the join or stir zone, which in turn must influence weld microstructure as well as consolidation of material in the root of the join. This study has been undertaken to investigate issues concerning heat generation, heat transfer and plastic deformation within the stir zone of friction stir welded aircraft aluminium alloys.
\end{abstract}

Key words: Friction stir welding; Backing bar; Aluminum alloys; Heat transfer.

\section{INTRODUÇÃO}

A utilização de ligas de alumínio nas indústrias automobilística e aeronáutica vem sendo amplamente explorada, principalmente por reduzir o peso de veículos e aeronaves - uma vez que a massa específica do alumínio é cerca de $1 / 3$ da massa específica do aço, sem que propriedades mecânicas e estruturais sejam comprometidas. Ainda, questões ambientais são fundamentalmente visadas diante da produção de ligas mais leves, econômicas e que geram menor quantidade de poluentes para a atmosfera. No entanto, por diversas vezes o emprego destas ligas torna-se inviável em função da dificuldade de unirem-se tais materiais.

\footnotetext{
'Aluno de Pós-graduação em Ciência e Engenharia de Materiais (PPGCEM) - UFSCar. Rodovia Washington Luís, km 235. Caixa Postal 676 13565-905 - São Carlos, SP, Brasil. mcuccolo@hotmail.com

2Professor Adjunto IV PhD do Dep. de Engenharia de Materiais (DEMa) da UFSCar. Rodovia Washington Luís, km 235. Caixa Postal 676 13565-905 - São Carlos, SP, Brasil.nelsong@ufscar.br

${ }^{3}$ Líder do grupo de soldagem do instituto GKSS Forschunszentrum GmbH. Joining Technology Group - Max-Planck-Str. I.D2 I 502 - Geesthacht, Alemanha.jorge.dos.santos@gkss.de
} 
Dado seu potencial de aplicação, novas alternativas de processamento e fabricação vêm se desenvolvendo, dentre os quais está o processo de soldagem no estado sólido, conhecido por Friction Stir Welding (FSW). Uma ferramenta cilíndrica não consumível, constituída por pino e ombro de geometrias definidas, caracteriza a solda por FSW. A solda é concretizada por esforços termomecânicos aplicados pela ação rotacional da ferramenta em contato e no interior da peça, em temperaturas abaixo do ponto de fusão do material.(I) A rotação da ferramenta e o contato do ombro com a peça promovem a geração de calor necessário para plastificar o material, que é movimentado ao redor do pino de modo que a solda seja consolidada na parte posterior da ferramenta. $O$ ombro, cuja direção é tipicamente normal ao eixo de rotação da ferramenta, está conectado ao pino de menor diâmetro, promovendo a maior parte do calor gerado e a concentração de material na superfície da junta.

A combinação dos parâmetros de processo, incluindo a velocidade de rotação e de avanço da ferramenta e força axial aplicada, é essencial para produzir uma solda de qualidade. Como o processo envolve a relação de escoamento e deformação plástica do material, pequenas variações, associadas à mudança nos materiais que compõem o backing, podem resultar na formação de defeitos volumétricos na stir zone (SZ) e alterar as condições de transferência de calor. ${ }^{(2)}$ Usualmente, o backing é confeccionado em aço e sua função principal é a de consolidar a solda na raiz, além de influenciar as zonas afetadas termomecanicamente, quanto a formato, perfil e tamanho. A condutividade térmica do backing faz com que o fluxo de calor extraído promova alterações metalúrgicas importantes no processamento de ligas de alumínio soldadas por FSW.

Tipicamente, os efeitos combinados entre a aplicação da força axial e a ação rotacional de fricção da ferramenta com o material dão origem à quase totalidade do calor gerado durante o processo de soldagem. ${ }^{(4)}$ Porém, devido ao elevado grau de deformação imposta pelo processo, regióes imediatamente adjacentes ao campo de atuação da ferramenta no centro da junta soldada também contribuem para geração e, consequente, transferência de calor. A condição de deformação plástica imposta pelo processo influencia na direção e no fluxo de calor gerado durante o escoamento do material.

\section{MATERIAIS E MÉTODOS}

As ligas de alumínio utilizadas compreendem as da série 2xxx e 6xxx, dos sistemas Al-Cu-Mg AA2024-T35I e Al-Mg-Si AA6013-T6, respectivamente. A escolha dos parâmetros de soldagem foi determinada por meio de estudos previamente realizados. $\mathrm{Na}$ primeira etapa, chapas de $4 \mathrm{~mm}$ da liga AA60I3-T6 foram soldadas sob duas combinações de parâmetros e três condições do backing (aço,cobre e cerâmico). Posteriormente, chapas de 3,2 mm da liga AA2024-T35I foram soldadas em três combinações de parâmetros. Em ambas as situações, as combinações dos parâmetros de soldagem (Tabela I) variaram quanto à velocidade de rotação da ferramenta e a velocidade de avanço da solda, mantendo-se constantes a força axial aplicada e a geometria da ferramenta (Figura I).

Tabela I. Combinação dos parâmetros de soldagem para as três condições de backing (cobre, aço e cerâmico) a uma força axial constante de $10 \mathrm{kN}$

\begin{tabular}{|c|c|c|c|}
\hline $\begin{array}{l}\text { Parâmetros } \\
\text { de Soldagem }\end{array}$ & $\begin{array}{l}\text { Velocidade } \\
\text { de Rotação } \\
\text { (RPM) }\end{array}$ & $\begin{array}{l}\text { Velocidade } \\
\text { de Avanço } \\
(\mathrm{mm} / \mathrm{min})\end{array}$ & $\begin{array}{l}\text { Liga de } \\
\text { Alumínio }\end{array}$ \\
\hline I & 900 & 600 & AA60I3-T6 \\
\hline 2 & 1.200 & 900 & $\begin{array}{l}\left(W_{p}=I, 50 e\right. \\
\left.W_{p}=I, 33\right)\end{array}$ \\
\hline 3 & 1.600 & 800 & AA2024-T35I \\
\hline 4 & 800 & 400 & $\left(W_{p}=2,0\right)$ \\
\hline 5 & 400 & 200 & \\
\hline
\end{tabular}

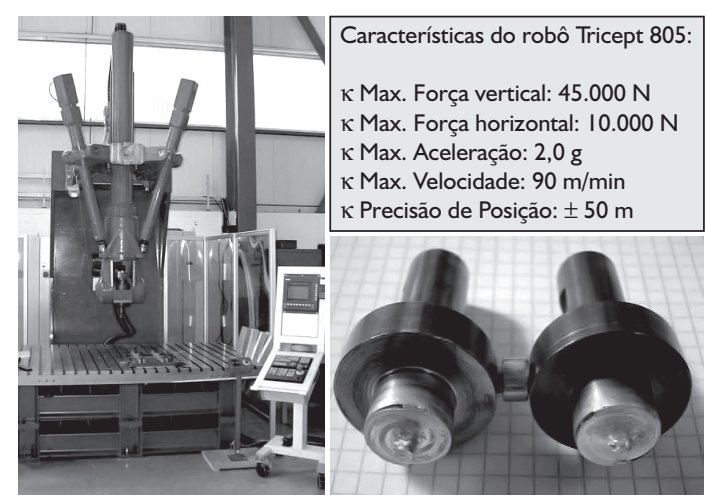

Figura I. FSW Robô Tricept 805 e ferramenta utilizada (pino facetado com roscas) (Fonte: GKSS).

As soldas por Friction Stir Welding foram produzidas no instituto alemão GKSS, em juntas de topo e bead on plate (BOP), que consiste em uma solda sem pino, somente ombro. Foi utilizado um robô Neos Tricept TR 805 (Figura I), capaz de mover-se em cinco eixos centrais, controlado por um sistema CNC de comando Siemens Sinumeric 840D. Ainda a força axial e a velocidade de rotação da ferramenta foram controladas por um sistema integrado, desenvolvido no próprio instituto.

A extração de calor no backing foi conduzida por materiais de condutividade térmica diferente: em um extremo cobre foi utilizado como condutor e, em outro, um material cerâmico como isolante térmico. Os backing bars utilizados na soldagem das ligas em estudo estão identificados na Figura 2. Uma mesa Horst Witte foi utilizada para gerar vácuo a fim de fixar as chapas soldadas na superfície e os backing, parafusados mecanicamente, no centro da linha de solda. 
Os dados de temperatura foram medidos em todas as soldas produzidas por meio de quatro termopares do Tipo-K posicionados estratégica e simetricamente em ambos os lados nas chapas soldadas (lado de avanço e retrocesso) a uma profundidade de $2 \mathrm{~mm}$ e $3 \mathrm{~mm}$ e a uma distância de $10 \mathrm{~mm}$ e $12 \mathrm{~mm}$ do centro da solda e, ao longo do eixo central nos backing bars, a uma profundidade de $0,5 \mathrm{~mm}$ da interface entre a chapa e a superfície do backing (Figura 3). Os dados de temperatura foram avaliados por meio de um microcomputador integrado ao sistema de aquisição de dados National Instrument SCXI-1000.

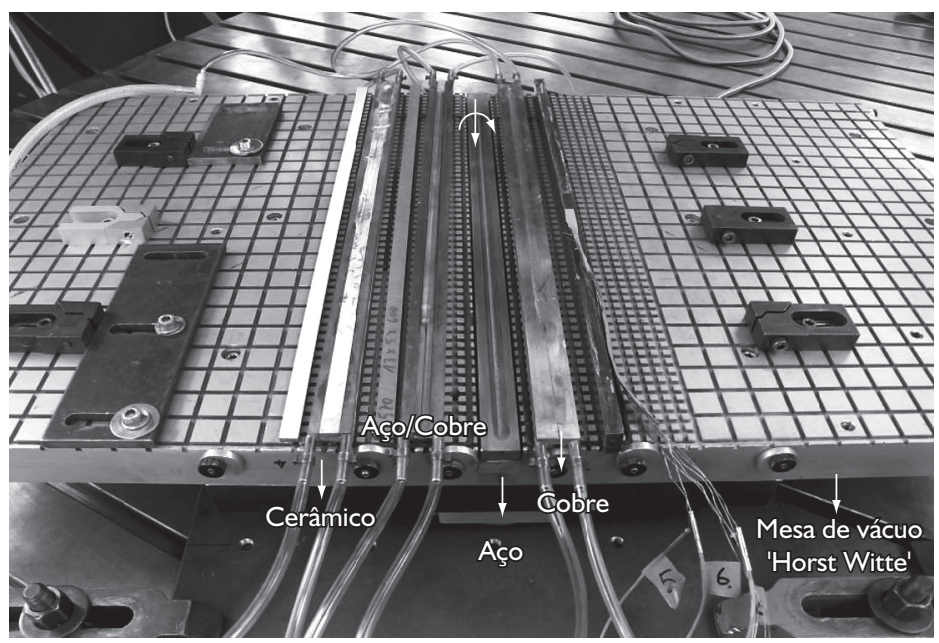

Figura 2. Backing de diferentes materiais utilizados durante o processo FSW sob a mesa com sistema de fixação mecânica e a vácuo das chapas soldadas (Fonte: GKSS).
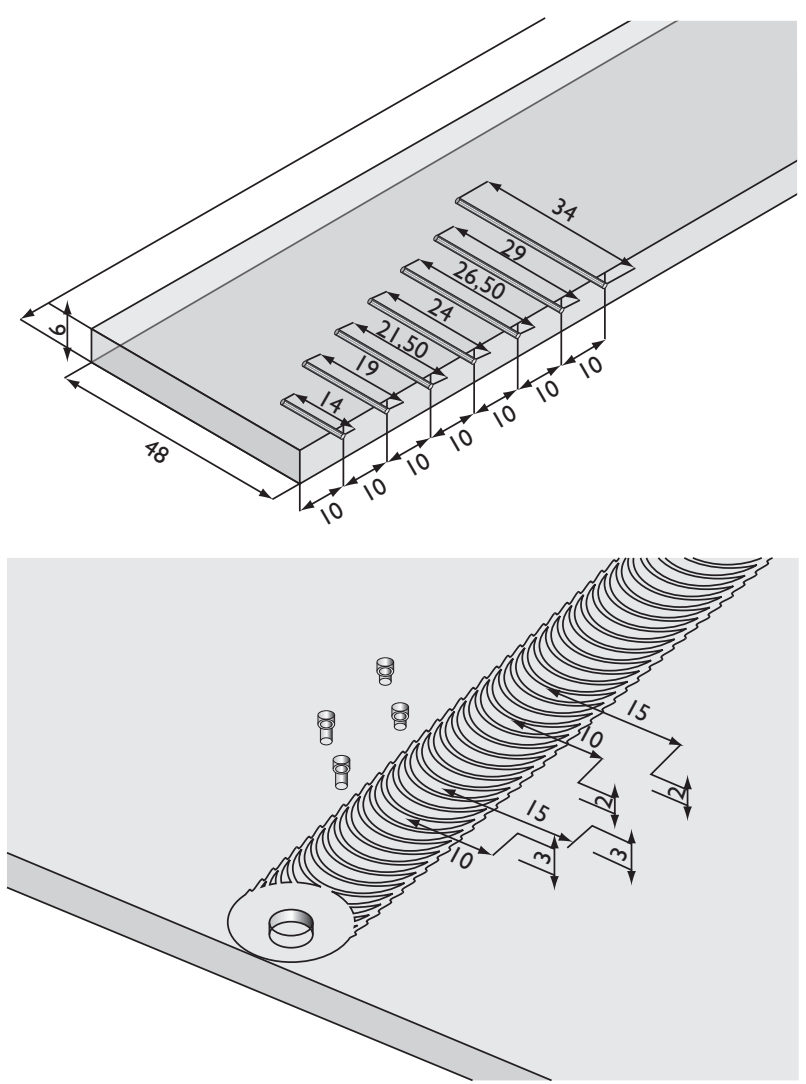

(b)

Figura 3. Posição dos termopares: a) no backing e b) nas chapas de alumínio.
A caracterização macro/microestrutural das regiões soldadas foi realizada pela técnica de microscopia óptica, com o objetivo de avaliar as avaliação das transformações metalúrgicas ocorridas após o processo de soldagem, e as mudanças de perfil, tamanho e geometria das zonas deformadas plasticamente. Medições de microdureza foram determinadas para traçar os perfis das amostras soldadas (em relação à distância do centro da solda) em cada condição de backing, a fim de se avaliar a influência dos três materiais (cobre, aço e cerâmico) nas propriedades mecânicas da junta.

\section{RESULTADOS E DISCUSSÕES}

Analisando os resultados obtidos das soldas FSW na liga 2024-T35I é evidente que a interação mecânica entre a ferramenta e o material influencia significativamente o tamanho da SZ. Com o aumento da rotação da ferramenta, há um decréscimo na área da $\mathrm{SZ}$ formada, pois a deformação ocorre bem próxima à ferramenta, uma vez que as ligas da série $2 x x x$ possuem elevada resistência à deformação plástica. Isso não ocorre devido a temperaturas mais baixas de processamento (Figura 4), mas porque uma porção maior do calor gerado é transferido verticalmente do material para a ferramenta FSW ao invés de ser disperso transversalmente ao longo da solda.

A exceção observada ocorreu para soldas realizadas com backing de cobre. Na realidade, a menor área de SZ medida foi criada com menor parâmetro de velocidade de rotação da ferramenta (400 RPM - $200 \mathrm{~mm} / \mathrm{min}$ - $10 \mathrm{kN}$ ) combinado com o uso do backing de cobre. Deve ser notado, no entanto, que nesta instância houve a fratura do pino na base do ombro. Isso ocorreu devido a uma temperatura insuficiente para amolecer o material e mais ainda porque sobre tal condição foram impostas forças cisalhantes no pino.

Os perfis de microdureza e a análise microestrutural mostram que a SZ é mais mole que o metal base quando soldado com backing de aço e cerâmico, em comparação com o de cobre. Os valores de dureza aumentam em para o caso do backing de cobre em função das baixas temperaturas de processo e também devido ao aumento da extração de calor do backing (aproximadamente 6 vezes mais quando comparado com o backing de aço). Como consequência, há energia e tempo insuficientes para ativar a degradação da microestrutura, como ocorrem casos dos backing de aço e cerâmico. 


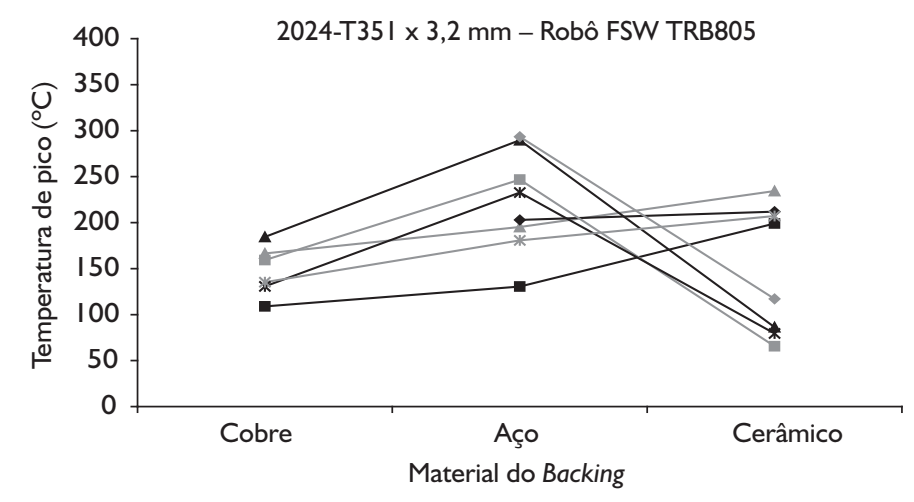

\begin{tabular}{|c|c|}
\hline-1600 RPM-800 mm/min(Plate) & $\triangle-800$ RPM-400 mm $/ \mathrm{min}$ (Plate) \\
\hline$\rightarrow-400 R P M-200$ mm/min(Plate) & - $1600 \mathrm{RPM}-800 \mathrm{~mm} / \mathrm{min}$ (B.bar) \\
\hline$\star 800$ RPM-400 mm/min(B.bar) & $\rightarrow-400$ RPM-200 mm/min(B.bar) \\
\hline * 800 RPM-400 mm/min(B.O.P.)B.bar & * $800 \mathrm{RPM}-400 \mathrm{~mm} / \mathrm{min}$ (B.O.P.)plate \\
\hline
\end{tabular}

Figura 4. Temperatura máxima registrada nas chapas de alumínio da liga AA 2024-T35 I e no backing em função do tipo de material que compõe o backing. Para o menor parâmetro soldado com backing de cobre não foram registrados dados de temperatura.

É interessante ressaltar, no entanto, que nos perfis de microdureza ao longo de todas as condições de backing (Figura 5), a largura das zonas termicamente afetadas, transversal à solda, em comparação com a direção de avanço da solda, mantém-se relativamente inalterada. Essa largura aproxima-se ao diâmetro do pino, o que indica uma condição de escorregamento na interação ferramenta/material sobre todas as condições de soldagem para essa liga. Similarmente, a microestrutura confirma a evidência de defeitos volumétricos na SZ.
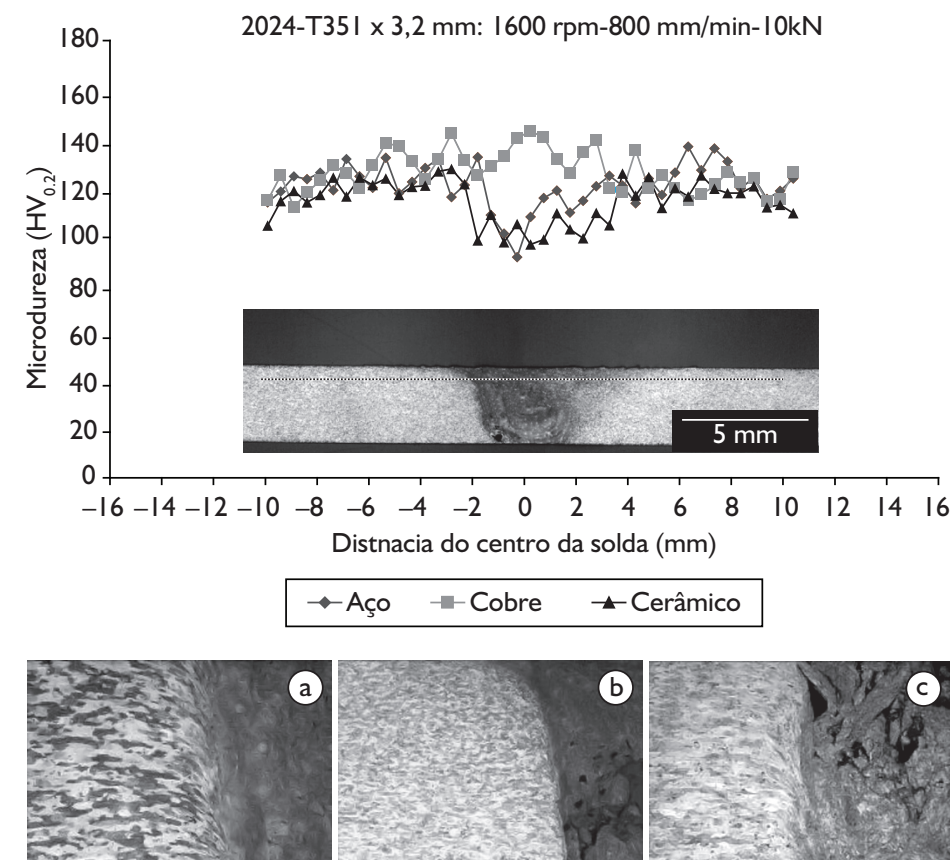

Figura 5. Perfiss de microdureza para a liga AA 2024-T35 I soldada por FSW e microestruturas das três condições de backing. a) aço; b) cobre; e c) cerâmico, com evidência de defeitos volumétricos formados na raiz da solda.
As soldas produzidas confirmam que a liga 60I3-T6 é facilmente deformada durante o processo FSW com a maior parte da deformação produzida devido ao contato do ombro. Quanto ao tamanho das SZ, verifica-se uma variação significativa em função do tipo de material que compõe o backing. Nota-se também, para este caso, que a área da SZ decresce com o aumento da velocidade de rotação da ferramenta.

A transferência de calor durante o processo é afetada diferentemente quando comparada com a das soldas na liga 2024-T35I, em que o backing exerce a função de extração de calor de forma mais intensa. Assim, para a liga 60/3-T6, um aumento da SZ só é possível sob uma condição adesiva de interação entre o material e a ferramenta (pino e ombro). Ainda, com o uso de material cerâmico, o bloqueio da transferência de calor para o backing força o calor a ser dissipado radialmente para o material, como evidencia a Figura 6.

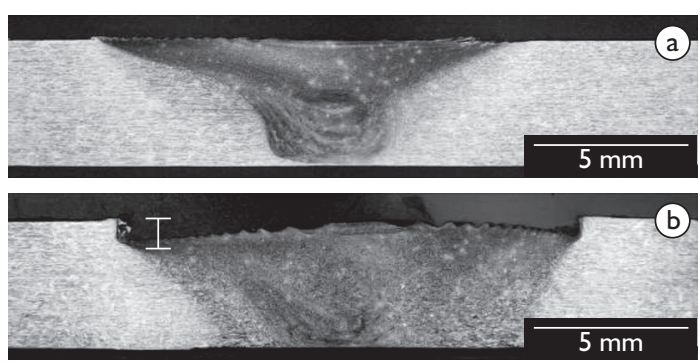

Figura 6. Macrogrtafia transversal da solda na liga AA 6013-T6: I) com backing de aço e 2) backing cerâmico, maior contato do ombro e maior área da SZ (900RPM $600 \mathrm{~mm} / \mathrm{min}-10 \mathrm{kN})$.

A relação entre a temperatura medida no material e a geometria da SZ é fortemente evidente para liga 60I3-T6. Quando a transferência de calor é reduzida no backing nota-se que maiores temperaturas de processamento induzem a formação de grandes áreas da SZ, conforme apresentado na Figura 7.

É evidente que nas soldas produzidas com o backing cerâmico há maior degradação da microestrutura e, em consequência, uma queda das propriedades mecânicas, evidenciada pelos perfis de microdureza (Figura 8). Alem disso, as ZTA e ZTMA também são influenciadas de modo que os valores de dureza são geralmente menores quando comparados com outra condição de backing (cobre). Os perfis medidos para o backing de aço são típicos da série $6 x x x-T 6$ quando soldado por FSW. A SZ é consideravelmente mais mole que o metal base, porém mais duro que a ZTA e ZTMA, uma vez que os precipitados estão dissolvidos e dinamicamente recristalizados. $\mathrm{Na}$ condição do backing de cobre é observado um decaimento mais acentuado da dureza devido à mudança da transferência de calor, que é direcionada para o material. 


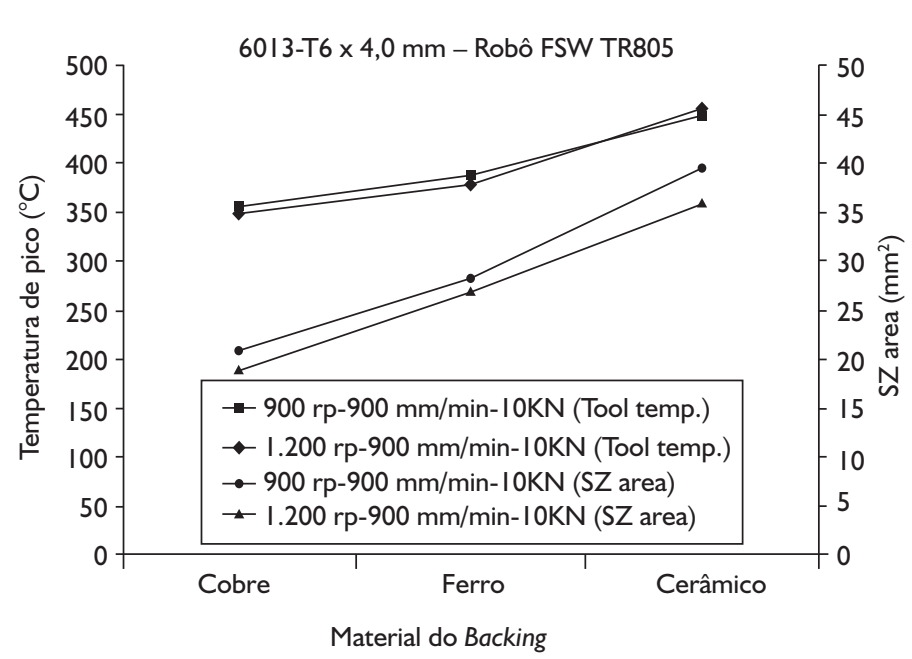

Figura 7. Temperatura máxima da ferramenta e área da $\mathrm{SZ}$ em função do material do backing. Temperaturas mais elevadas foram registradas também nas chapas da liga AA 60I3-T6 quando o calor é bloqueado no backing (cerâmico), o que leva à interpretação de instabilidade térmica do processo FSW.

6013-T6 x 4 mm: $1200 \mathrm{rpm}-900 \mathrm{~mm} / \mathrm{min}-10 \mathrm{kN}$
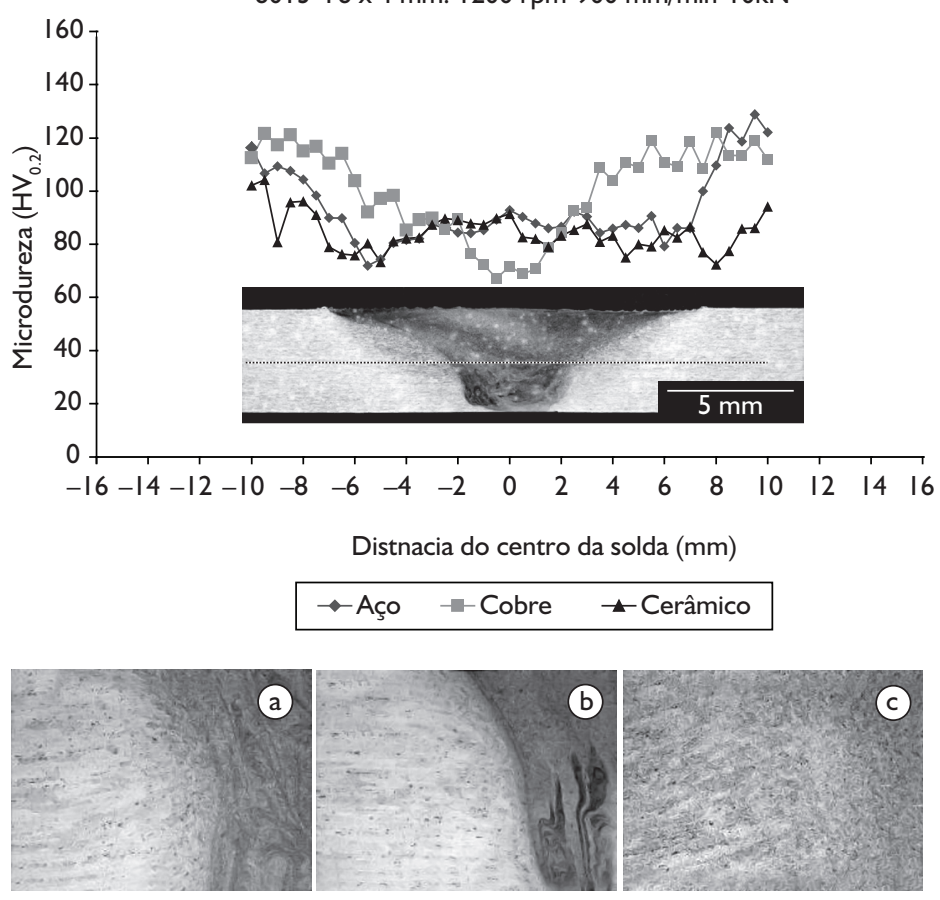

Figura 8. Perfis de microdurezas da liga AA 60/3-T6 soldada por FSW e microestruturas das três condições de backing: a) aço; b) cobre; e c) cerâmico.

\section{CONCLUSÕES}

Este estudo apresenta evidências claras que a condição de transferência de calor difere de acordo com o tipo de liga de alumínio (composição química), com os parâmetros de processo e com a condutividade térmica do backing durante a soldagem pelo processo FSW. As soldas produzidas indicam que uma condição de deformação na interface entre o material e a ferramenta domina o modo de transferência de calor durante a soldagem.

A influência da transferência de calor nas propriedades mecânicas e nas características microestruturais de juntas soldadas por FSW é atribuída à resistência à deformação plástica do material. A principal fonte geradora de calor é a deformação imposta pelo processo, nas regiões imediatamente adjacentes ao campo de atuação da ferramenta no centro da junta soldada. A causa da interação mecânica material/ferramenta ocorre sob uma condição de escorregamento, para ligas da série $2 x x x$ ou adesão, para as da série $6 x x x$.

Assim, em ligas de elevada resistência à deformação (2024-T35I), a transferência de calor tem menor relevância durante o processo FSW. Isso ocorre porque, com $\circ$ ajuste e a seleção apropriada de parâmetros (essencialmente velocidades de avanço e de rotação da ferramenta), é possível garantir soldas de qualidade.

Este não é o caso, no entanto, das ligas de baixa resistência à deformação e artificialmente envelhecidas, como as do sistema Al-Mg-Si (60I3-T6). Devido a uma condição de adesão entre a ferramenta e o material, a utilização do backing de cobre promove maior deslizamento do material na interface da solda, o que garante estabilidade térmica ao processo para soldas realizadas nessas ligas.

\section{Agradecimentos}

Ao apoio financeiro e suporte técnico do instituto de pesquisas alemão GKSS Forschunszentrum, um dos centros mundiais de referência em soldagem robotizada por FSW, especialmente em nome do Dr. Rudolf Zettler.

Ao Departamento de Engenharia de Materiais (DEMa) e ao Centro de Caracterização e Desenvolvimento de Materiais (CCDM) da Universidade Federal de São Carlos.

\section{REFERÊNCIAS}

I Mishra, R.S.; Ma, Z.Y. Friction stir welding and processing. Materials Science and Engineering, v. 50, n. I-2, p. I-78, Ago. 2005.

2 Zettler, R.; Lomolino, S.; Santos, J.F; Donath, T.; Beckmann, F.; Lippman, T.; Lohwasser, D. A study on material flow in FSW of AA 2024-T35I and AA 6056-T4 alloys. In: INTERNATIONAL FSW SYMPOSIUM, 5., 2004, Metz, França. [S.n.t.]. p. I-13.

3 ULYSSE, P. Three-dimensional modelling of the friction stir-welding process. International Journal of Machine Tools \& Manufacture, v. 42, n. 14, p. 1549-57, Sept. 2004. 
4 Russell, M. J.; Schercliff, H.R. Analytical modelling of microstructure development in friction stir welding. In: INTERNATIONAL FSW SYMPOSIUM, I. I999, Thousand Oaks. [S.I.: s.ed.], 200 I. p. I-I I.

5 Colegrove, P. 3 Dimensional flow and thermal modelling of the friction stir welding process. In: INTERNATIONAL FSW SYMPOSIUM, 2., 2000, Stockholm, Suécia. [S.n.t.]. p. I-II.

6 Lienert, T.J.; Stellwag, W.L.; Lehman, L.R. Heat inputs, peak temperatures and process efficiencies for FSW. In: INTERNATIONAL FSW SYMPOSIUM, 4., 2003, Utah. [S.n.t.]. p.I-I0.

7 Vilaça, P.; Quintino, L.; Santos, J.F.; Zettler, R.; Sheikhi, S. Quality assessment of friction stir welding joints via an analytical thermal model. Materials Science and Engineering A, v. 445-6, p. 50I-8, Febr. 2007.

Recebido em: 22/10/2008

Aceito em: 27/02/2009

Proveniente de: CONGRESSO ANUAL DA ABM, 63., 2008, Santos, SP. São Paulo: ABM, 2008. 